\title{
summary
}

\section{Decision analysis in dentistry}

Rohlin M, Mileman PA. Decision analysis in dentistry - the last 30 years. J Dent 2000; 28:453-468

Objective To review publications in dentistry of decision analyses during the last 30 years.

Data sources Medline 1969-1998 and hand-searching of reference lists of retrieved publications.

Study selection Articles presenting a decision analysis with utilities and sensitivity analyses were included.

Data extraction and synthesis A quality assessment of the included studies but no formal synthesis due to the range of clinical applications considered.
Results Sixty-seven articles were identified with 22 fulfilling the criteria for inclusion.

Conclusions Decision analyses publications in dentistry have declined in recent years. The quality of decision analyses in dentistry is variable. Improvement in this promising technique will depend on improvement in the quality of basic research data.

Address for reprints: $M$ Rohlin, Department of Oral Radiology, Centre for Oral Health Sciences, Carl Gustafs vag 34, 21421 Malmö, Sweden.

E-mail: madeleine.rohlin@od.mah.se

\section{Commentary}

This paper is a systematic review of decision analyses in dentistry. Decision analysis is a method that complements the randomised control trial (RCT) or, in some circumstances, it acts as an alternative to the RCT. It is particularly useful for problems where the issues are complex, where an RCT would be unethical or in circumstances where the current information is uncertain. ${ }^{1}$ The paper utilises a methodology directly analogous to systematic review of RCTs in order to examine the decision-analysis literature. Importantly, it utilises quality scoring of the primary literature, which is a key element of a truly systematic review. The authors do admit, however, that they found application of their quality-scoring criteria rather difficult and, as with many of the taxonomies utilised in RCT quality-scoring, it would not be difficult to argue that the criteria used were neither mutually exclusive of each other nor were they exhaustive. Nevertheless, it is to be hoped that others might however build on this important first contribution to the literature and further develop the means by which decision analysis papers may be systematically assessed.

The paper clearly shows that decision analysis in dentistry is under-utilised and often poorly applied. Why this should be so is unclear. Given that the technique provides the key to realistic cost-effectiveness analysis, the authors reveal that the research 'skill base' in dentistry requires development, in the field of decision analysis. The paper demonstrates that of the 22 articles presenting decision trees, only 15 made clear to the reader the basic assumptions underpinning the analysis. Even more disappointing is the revelation that only two papers had included patient-based utility values. In an era where patient sovereignty and the subjective nature of health are increasingly being recognised, ${ }^{2}$ this is a startling finding and contrasts dramatically with trends in medicine. Perhaps the most important observation in this paper is that the evidence base in some areas of dentistry is so sparse that it is virtually impossible to undertake decision analysis. That is, we currently have insufficient information to even begin to examine what health 'outputs' are derived from the highly expensive and often difficult 'inputs' that are involved in oral care. Thus, this paper shows that dentistry has not built its evidence base to an extent whereby decision analysis can be universally used as a research tool. Furthermore, dentistry appears to have been slow to grasp and use the concept of utilities and subjective evaluation of health states. These omissions must be rectified before dentistry can truly be said to be evidence-based.

1. Petitti DB. Meta-analysis, decision analysis and cost-effectiveness analysis. Methods for Quantitative Synthesis in Medicine. Second Edition. New York: Oxford University Press, 2000.

2. Slade GD (Ed). Measuring Oral Health and Quality of Life. University of North Carolina, Chapel Hill, 1997.

\section{Elizabeth Kay Oral Health and Development, University of Manchester,} UK 